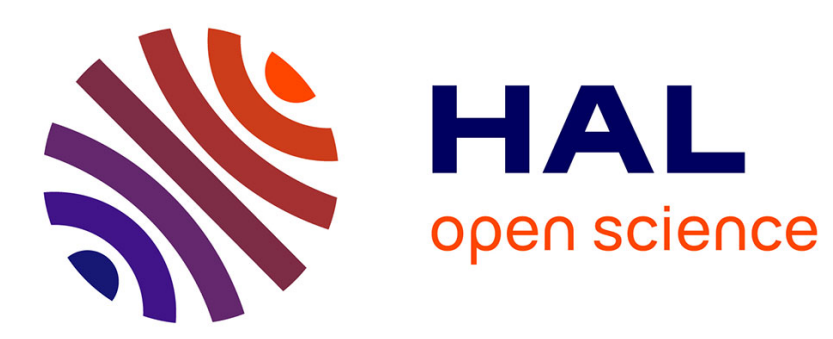

\title{
The development of Radon transforms associated to Compton scatter imaging concepts
}

\author{
Mai K. Nguyen, T. T. Truong
}

\section{To cite this version:}

Mai K. Nguyen, T. T. Truong. The development of Radon transforms associated to Compton scatter imaging concepts. Eurasian Journal of Mathematical and Computer Applications, 2018, 1 (1). hal01759845

\section{HAL Id: hal-01759845 \\ https://hal.science/hal-01759845}

Submitted on 5 Apr 2018

HAL is a multi-disciplinary open access archive for the deposit and dissemination of scientific research documents, whether they are published or not. The documents may come from teaching and research institutions in France or abroad, or from public or private research centers.
L'archive ouverte pluridisciplinaire HAL, est destinée au dépôt et à la diffusion de documents scientifiques de niveau recherche, publiés ou non, émanant des établissements d'enseignement et de recherche français ou étrangers, des laboratoires publics ou privés. 


\title{
The development of Radon transforms associated to Compton scatter imaging concepts
}

\author{
M. K. Nguyen* ${ }^{*}$ and T. T. Truong** \\ *Equipes de Traitement de l'Information et Systèmes (ETIS), \\ Université de Cergy-Pontoise/ENSEA/ UMR CNRS 8051 \\ F-95302 Cergy-Pontoise Cedex, France, \\ ** Laboratoire de Physique Théorique et Modélisation (LPTM), \\ Université de Cergy-Pontoise/ UMR CNRS 8089 \\ F-95302 Cergy-Pontoise Cedex, France.
}

\begin{abstract}
For more than three decades, the exploitation of Compton scattering has led to significant progress in imaging the inner parts of an optically opaque object. In this imaging process, the Compton scattering angle plays an essential role as it labels the recorded data, suppressing the motion of the scanning camera, as well as generates new geometric manifolds on which radiation measurements are made and thereby introducing new relevant Radon transforms. The present paper is aimed to synthetize the conceptual development of Compton scattering imaging in order to gain a constructive overview and possibly induce a further innovative growth to it. We believe this may lay foundation to new mathematical tools as well as lead to a great diversity of applications.
\end{abstract}

\section{Introduction}

Since the seminal work of A M Cormack in 1963 [1], the original Radon transform has become the major mathematical tool for imaging hidden features of an object. This is the case in medicine, non-destructive evaluation or control and in many other fields of research, inspection and exploitation. During this time, many new imaging processes have come up thanks to the advances in science and technology. Many of them have led to generalizations of the original Radon transform. Most importantly are generalizations that are due to the exploitation of the so-called Compton effect in physics. In this paper, we give an account of the development of imaging proposals whic have emerged from the use of the Compton effect in recent years and their underlying generalized Radon transforms.

The exposition adopted here will follow a historical path in order to show how ideas evolve in time and how breakthroughs are made in the course of research. To emphasize new aspects, we shall first recall the state of art of the current imaging processes using penetrating radiation, which comprises emission, transmission and reflection imaging (section 2). Then we reexamine the properties of the Compton effect in these imaging processes before introducing the concept of scattered radiation

$\dagger$ To whom correspondence should be addressed (mai.nguyen-verger@u-cergy.fr) 
imaging (section 3). In the next step, we exhibit some typical new Radon transforms that have emerged from these considerations, namely the Cone Radon transform (section 4) and its two-dimensional counter part, called the V-line Radon transform (section 5). Curiously we shall also discover later that V-line Radon transform is connected to a special modality of the so-called Compton Scatter Tomography (CST), which is in fact a transmission-scattering imaging process, an evolved form of the original Compton Scatter Tomography (CST) designed by S J Norton in 1995 [2]. We should recall that CST is also an alternate way of exploiting the Compton effect for imaging purposes. The next section 6 describes some main potential applications in diverse fields such as medical diagnostic, non-destructive material evaluation, nuclear safety monitoring, etc. illustrated by simulations. A conclusion will summarize the merits and challenges of the Cone and V-line Radon transforms in future developments of imaging science.

\section{Primary radiation imaging based on ray-matter interaction}

All of primary radiation imaging principles are based on rectilinear propagation and its interaction with traversed matter. This results in three main imaging processes by transmission, emission and reflection, as illustrated in Fig. 1.
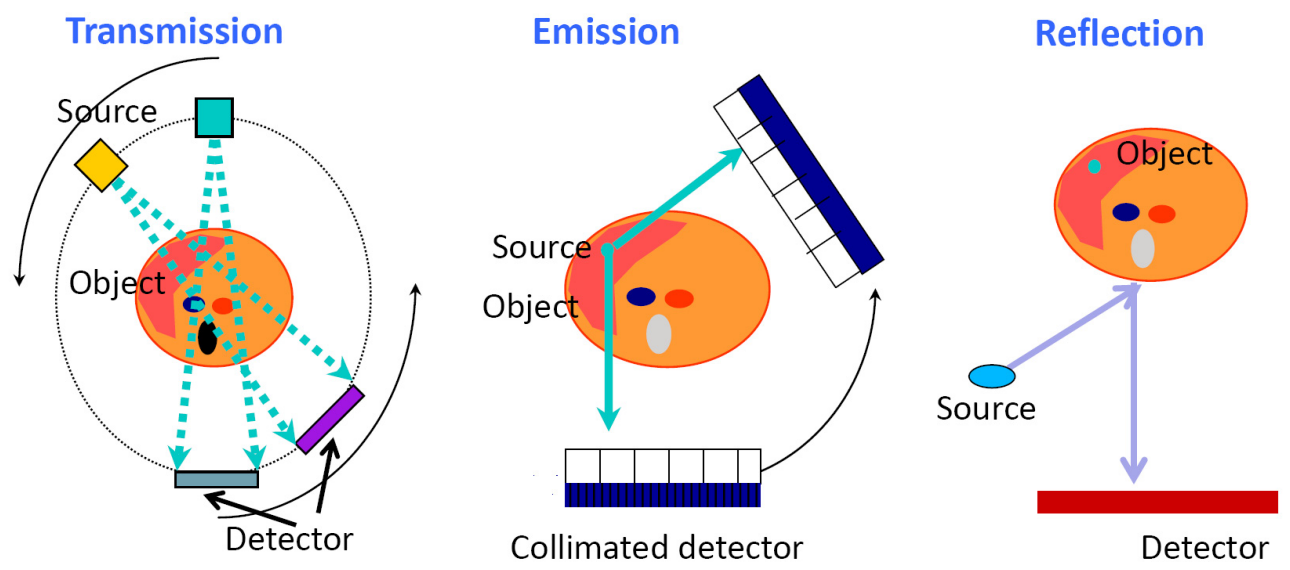

Figure 1. Primary radiation imaging modalities

Transmission imaging measures the values of integrated radiation attenuation along all rectilinear paths of propagation which cross an object. Attenuation is due to absorption and scattering of radiation in traversed matter. The complete set of these collected measured values may be represented as the classical Radon transform of the linear coefficient of attenuation of the studied object. As it is well-known, one may reconstruct this quantity using the inverse classical Radon transform.

In transmission imaging, the object is a passive medium. This object may be turned into an active emitting medium, for example by injection of a radioactive tracer which shall distribute itself non uniformly in the bulk of the object. Then by positioning outside the object a hole collimated detector, one may measure the emanating radiation flux along the line, which is the axis of the hole collimator. Then a complete set of such measurements may be again identified to the Radon transform 
of the density of radiation emitters in the object. This is called Single Photon Emission Tomography (SPECT).

Lastly, reflection imaging makes use of the reflectivity of the surfaces of object. It concerns mainly optical and(or) microwave radiation (radar). The third figure at right in Fig. 1 shows that there is a need of illumination by an external source, the detector may simply be a photographic plate.

By primary radiation imaging, we mean that the detected radiation has the same energy (or wavelength) as the original (or incident, or emitted) radiation. Image quality from primary ( $\mathrm{X}$ or gamma ray)-radiation imaging processes is usually affected by the phenomena of Compton scattering, and a lot of efforts is devoted to removing its de-gradating effects.

\section{Compton effect and its consequences in primary radiation imaging}

In this section we recall some properties of Compton scattering. Light scattering is a wide spread phenomena experienced by all of us. When we see a green line in the dark night sky arising from a "green" laser source, this is because the green light emitted by the laser strikes the air molecules of the atmosphere and gets reflected back to the observing eye. Such scattering is called elastic in the sense that this scattering does not change the wavelength after scattering.

However for higher energy radiation (such as $\mathrm{X}$ or gamma rays), this radiation does not collide with molecules but with electrons in matter and concurrently there is a change in energy (or wavelength) after scattering. This is the main feature in Compton scattering. In fact scattered radiation emerges with an energy $E(\omega)$ given by the so-called Compton formula

$$
E(\omega)=E_{0} \frac{1}{1-\epsilon \cos \omega},
$$

where $\omega$ is the scattering angle (see Fig. 2), $E_{0}$ the incident radiation energy, $\epsilon=E_{0} / m c^{2}$ and $m c^{2}$ the electron rest energy.

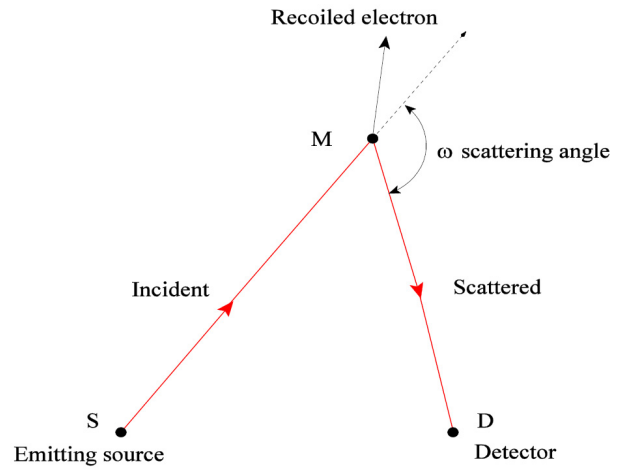

Figure 2. Compton scattering

We now understand why Compton scattering contributes to the attenuation of a traveling radiation beam as well as why it create blurs on images, see left figure of Fig. 3. Yet, one may take a resolutely opposite point of view by taking into account the effects of Compton scattering to improve quality in gamma-graphic images, as done in 
[3], (see right figure of Fig. 3), or more drastically consider the possibility of turning the Compton effect into a real effective imaging agent.
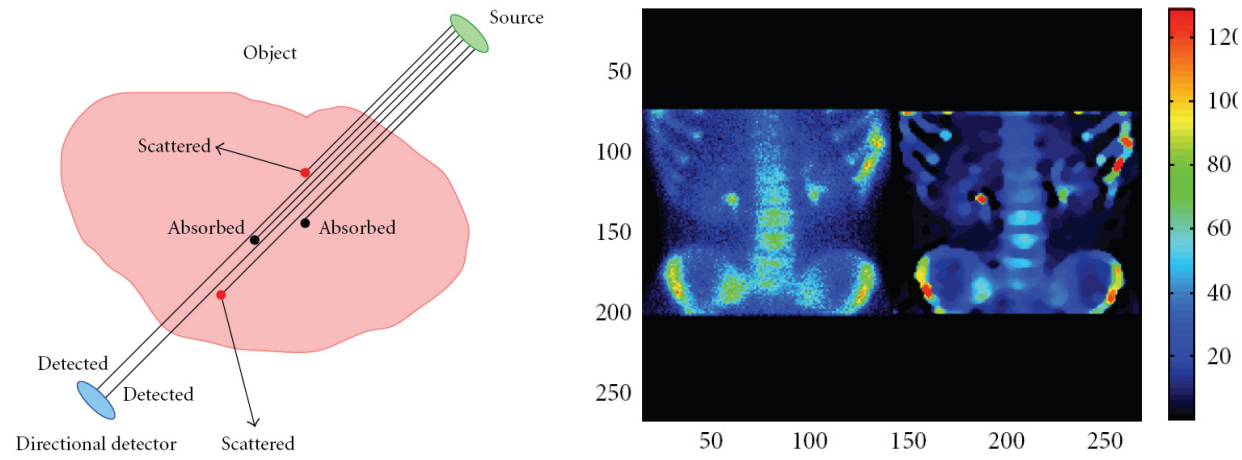

Figure 3. Left - Radiation attenuation, reprinted from $[10]$

Right - Bone scintigraphy: a scintigraphic image is restored without (left) and with (right) the use of Compton-scattered radiation (Hot spots or nodules are clearly displayed), reprinted from [3])

\section{Three-dimensional Compton scattered radiation imaging and various cone Radon transforms}

Despite the negative aspects presented by the Compton effect, there are attempts to use it for imaging the inner parts of objects as early as 1976 [4]. As can be seen from the dynamics of the scattering, the image given by such Compton scatter tomography would be the map of the electron distribution in the bulk of the objet under study. The development of this idea has gone through decades of work and is reviewed in [5]. For the moment we leave this topic for a later discussion (see section 6) and concentrate on another aspect of the utility of Compton scattering in imaging processes.

In 1983, M Singh has suggested to use the Compton effect to create a so-called electronic collimation to improve SPECT imaging sensitivity [6], see left figure of Fig. 4. This idea consists in letting primary radiation emitted by a radiating object Compton interact with a scattering planar medium (called scattering detector). Scattered radiation is then detected by coincidence by another planar detector, called absorption detector. The amount of scattered radiation collected at energy $E(\omega)$ and the line connecting the scattering site to the absorption site form the data which give the locations of all corresponding emitting source sites. It can be shown that all the emitting sites of the object contributing to one such measurement are located on a circular cone with vertex on the scattering detector, with opening angle $\omega$ and with symmetry axis the line connecting the scattering site to the absorption site. Therefore this set of conical projections, as opposed to linear projections encountered in primary radiation imaging, leads naturally to the notion of Radon transform on circular cones in $\mathbb{R}^{3}$. However a circular cone in $\mathbb{R}^{3}$ with vertex on a plane has up to five parameters and the function to be reconstructed has only three variables. Thus the problem of reconstructing the density of radio-activity emitters in $\mathbb{R}^{3}$ has remained a challenge for decades. Recently progress has been made in this direction by many workers who arrive at inversion formulas using full data (see section 4). 
About two decades ago, many attempts have been made to simplify the data formed by the conical projections. One of them came from the work of M J Cree and $\mathrm{P}$ J Bones [7], in which, for the problem to be manageable, one still keeps an absorption detector with vertical collimated holes in a direction perpendicular to the plane of the scattering detector, see right figure of Fig. 4. The set of relevant cones is now cones with vertex on the scattering detector, with opening angle $\omega$ and vertical symmetry axis, hence only with three parameters. However, no inverse formula has been established then.
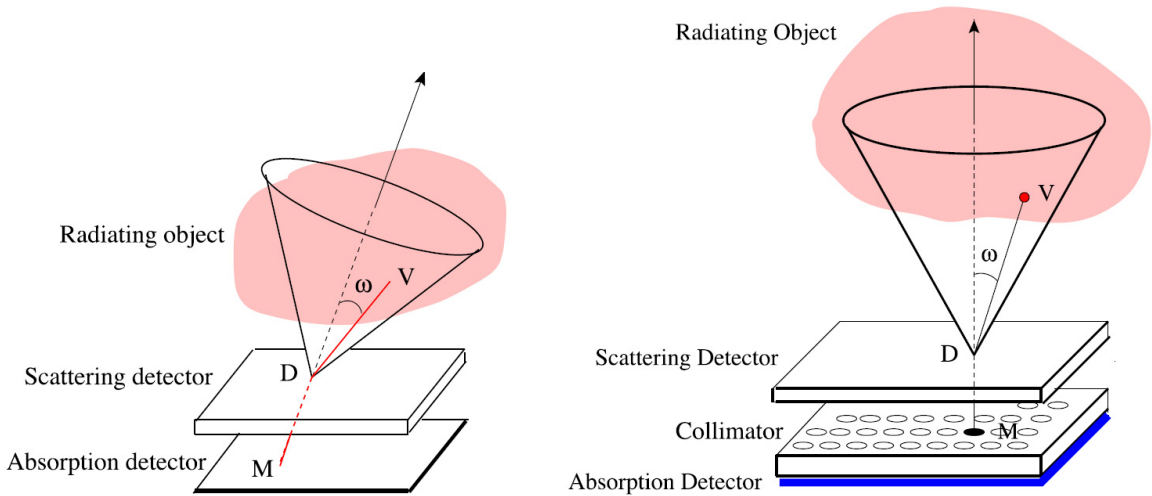

Figure 4. Compton camera (left) and Cree-Bones Compton camera (right), reprinted from [37]

In 2002, reconsidering the set up of $\mathrm{M} \mathrm{J}$ Cree and $\mathrm{P} \mathrm{J}$ Bones, but removing the scattering detector, we realized, that the counts at each site of the collimated absorption detector and at a single scattered energy $E(\omega)$, have a very interesting meaning. Take a site on the vertical line of a collimator hole, then this site may be considered to be a Compton scattering site for all emitting sites of the object situated on a circular cone admitting this vertical line as symmetry axis and having opening angle $\omega$. Thus the registered radiation counts at the absorption detector is due to the contributions of the sum of a set of identical "parallel" circular cones whose vertices are lined up on the axis of each collimator hole, see Fig. 5.
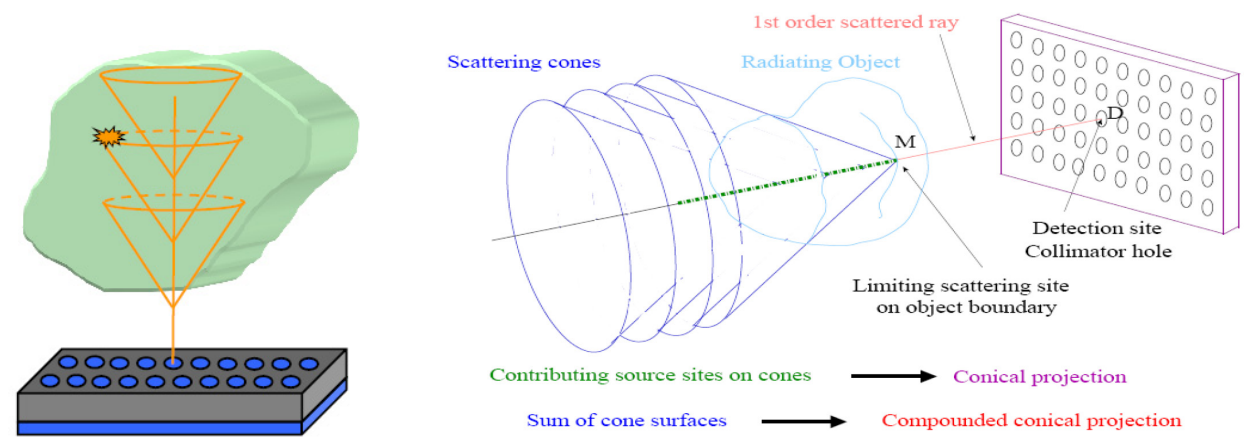

Figure 5. Compounded Conical Radon Transform, reprinted from [9]

In [8], we have called such a mapping of the object electron density a compounded 
conical Radon transform (CCRT). Note that each of such compounded conical projection has precisely three parameters: the two coordinates of the vertex on the collimated detector and the scattering angle $\omega[9,10]$. Hence it may be used, through its inverse, when it exists, to reconstruct the object electron density. We have succeeded to obtain the inverse formula and have performed numerical simulations to demonstrate the viability of this imaging process. The upshot of this new scattered radiation imaging is that one obtains a direct three dimensional image without having to rotate the detector as it was the case in conventional SPECT imaging. In short, we may say that the data obtained by physical rotation of the detector is replaced by the data at different scattering angle, in other words the physical rotation angle by the scattering angle. The fact that the scattered radiation detector remains motionless represents an enormous advantage in comparison with existing imaging modalities, since one is now free from the heavy and cumbersome mechanical rotation mechanism installed with the gamma camera. This imaging process may be given the name of Compton scatter SPECT imaging (CS-SPECT).

In the course of time, we have also realized that one piece of the Compounded Conical Radon Transform, which is the conical Radon transform considered by M $\mathrm{J}$ Cree and $\mathrm{P} \mathrm{J}$ Bones in their particular Compton camera may be exploited in transmission-scattering imaging. In the mean time, this conical Radon transform has led to an affiliated conical Radon transform, which has appeared recently in the literature $[12,13,14]$. Two possibilities arise with when the object is illuminated by a parallel beam, for example coming out from a synchrotron radiation accelerator, In Fig. 5 on the left, incident radiation from a parallel beam falls on an object and at a site of a detector is recorded scattered radiation with scattering angle $0<\omega<\pi / 2$ whereas on the right Compton scattering at an angle $\pi / 2<\omega<\pi$ (or backscattering) is realized by letting in incident parallel beam through a hole in the detector plane. The measured data is thus formed by these special conical projections with a fixed direction of the scanning cones. They may be fed into the inversion formula (see [15] to reconstruct the object electron density. As we shall see, the second procedure with back scattering turns out to be relevant for cultural heritage research imaging [38, 39], (see section 6).

To formalize the intuitive ideas, exposed above, we reproduce some theoretical steps on Cone and Compounded Cone Radon transforms, denoted successively by $\mathcal{R}_{C}$ and $\mathcal{R}_{C C}$.

- In Fig. 7, a site $S$ on a circular cone of vertical axis, with vertex $N=$ $\left(x_{N}=x_{D}, y_{N}=y_{D}, z_{N}=\zeta\right)$, opening angle $\omega$ will have Cartesian components $\left(x_{S}=x_{D}+r \sin \omega \cos \phi, y_{S}=y_{D}+r \sin \omega \sin \phi, z_{S}=\zeta+r \cos \omega\right)$, where $\phi$ is the azimuthal angle of $S$. Thus the cone integral of a function $f(x, y, z)$ with compact support may be always brought to the form

$$
\begin{gathered}
\mathcal{R}_{C} f\left(x_{D}, y_{D}, \omega \mid \zeta\right)= \\
K(\omega) \int_{\mathbb{S}^{1}} \int_{\mathbb{R}^{+}} r \sin \omega d \phi d r g(r) f\left(x_{D}+r \sin \omega \cos \phi, y_{D}+r \sin \omega \sin \phi, \zeta+r \cos \omega\right),(2)
\end{gathered}
$$

where $r=S N, r \sin \omega d \phi d r$, the cone integration measure, $g(r)$ is a function describing physical processes such as photometric effect $g(r)=1 / r^{2}$ or constant attenuation $g(r)=e^{-\mu r}$, or the combination of the two effects and $K(\omega)$ is the kinematic factor of the Compton effect proportional to the differential cross-section. 

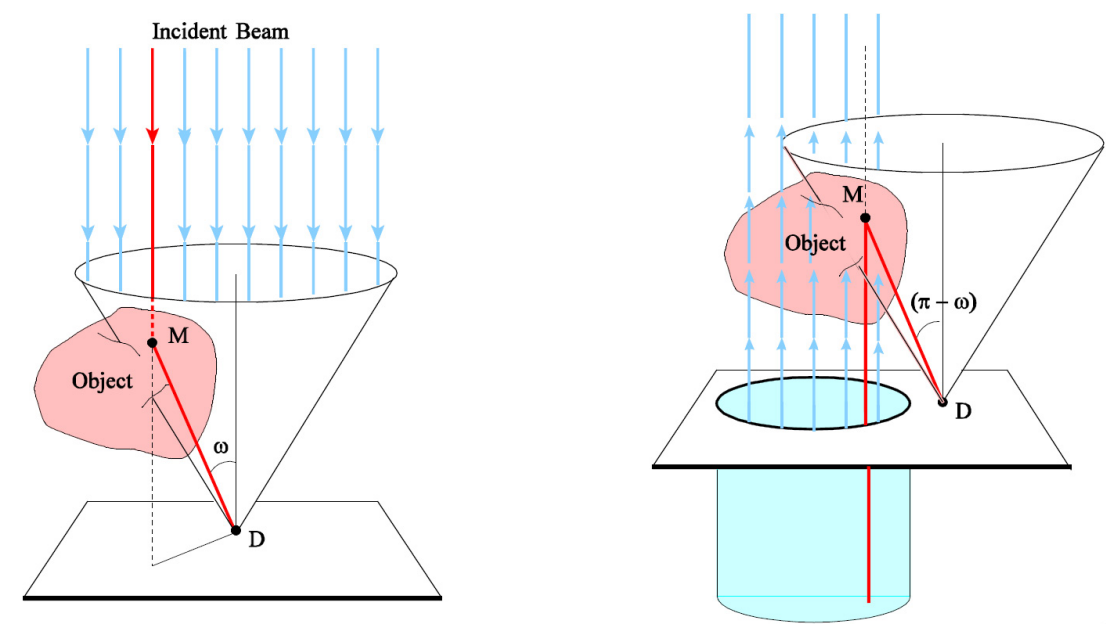

Figure 6. Three dimensional Transmission-Scattering Imaging with incident parallel beam

Equation (2) can be put under the form of an integral transform

$$
\mathcal{R}_{C} f\left(x_{D}, y_{D}, \omega \mid \zeta\right)=\int_{\mathbb{R}^{3}} d x d y d z \mathcal{K}_{C}\left(x_{D}, y_{D}, \omega \mid x, y, z\right) f(x, y, z),
$$

where the kernel $\mathcal{K}_{C}\left(x_{D}, y_{D}, \omega \mid x, y, z\right)$ is given by

$$
\mathcal{K}_{C}\left(x_{D}, y_{D}, \zeta, \omega \mid x, y, z\right)=K(\omega) g\left(\frac{\rho}{\sin \omega}\right) \delta((z-\zeta) \sin \omega-\rho \cos \omega),(4)
$$

where $\rho=\sqrt{\left(x-x_{D}\right)^{2}+\left(y-y_{D}\right)^{2}}$, displaying manifest translational invariance in the $x O y$ plane. For given $\left(x_{D}, y_{D}, \zeta, \omega\right)$, the vanishing of the delta function argument is just the equation of a circular cone of vertex at $\left(x_{D}, y_{D}, \zeta,\right)$ with opening angle $\omega$ in $(x, y, z)$ space. Conversely, for given $(x, y, z, \omega)$, this is the equation of a circular cone of vertex at $(x, y, z)$ and opening angle $\omega$ in $\left(x_{D}, y_{D}, \zeta,\right)$ space.

- For the Compounded Conical Radon Transform, we have the same structure

$$
\mathcal{R}_{C C} f\left(x_{D}, y_{D}, \omega\right)=\int_{\mathbb{R}^{3}} d x d y d z \mathcal{K}_{C C}\left(x_{D}, y_{D}, \omega \mid x, y, z\right) f(x, y, z),
$$

except that the kernel has a further integration on $\zeta$ with $d \zeta g(\zeta)$, namely

or

$$
\mathcal{K}_{C C}\left(x_{D}, y_{D}, \omega \mid x, y, z\right)=K(\omega) \int_{\mathbb{R}^{+}} d \zeta g(\zeta) g\left(\frac{\rho}{\sin \omega}\right) \delta((z-\zeta) \sin \omega-\rho \cos \omega),(6)
$$

$\mathcal{K}_{C C}\left(x_{D}, y_{D}, \omega \mid x, y, z\right)=H\left(\frac{z \sin \omega-\rho \cos \omega}{\sin \omega}\right) \frac{K(\omega)}{\sin \omega} g\left(\frac{\rho}{\sin \omega}\right) g\left(\frac{z \sin \omega-\rho \cos \omega}{\sin \omega}\right)$

where $H(z)$ is the Heaviside unit step function. More details can be found in [11], in particular for $g(r) \sim r^{-2}$ this kernel has the shape of a "Mexican hat".

We now give the expressions of the inverse transforms, which are used in the reconstruction of the unknown density $f(x, y, z)$. 


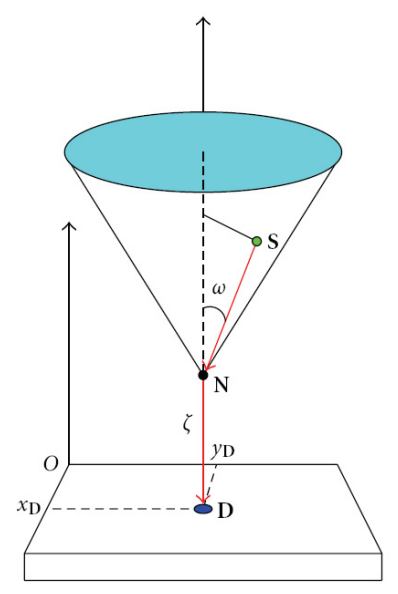

Figure 7. Coordinates for the Compounded Conical Radon Transform

- In deriving the inverse formula of the Cone Radon Transform $\mathcal{K}_{C}$, we observe that the set of complete data for reconstruction may consist of the scanning data with $0<\omega<\pi / 2$ or alternatively with $\pi / 2<\omega<\pi$, in other words we may operate with forward scattering or with backward scattering. These two theoretical possibilities are in full agreement with the two imaging processes given in Fig. 4. We also note that an analytical inverse can be obtained only for $g(r)$ proportional to a power of $r$.

The derivation of $\mathcal{K}_{C}^{-1}$ follows the steps of [15] by transforming equation (2), using polar coordinates, into a product of Hankel transforms of order zero and order $l$ for the $l^{\text {th }}$ of the unknown function. Let us define $F(\rho, \theta, z)=f(x, y, z)$, with $(x=\rho \cos \theta, y=\rho \sin \theta, z=z)$. Now let $\widetilde{f}(u, v, z)$ be the two dimensional Fourier transform of $f(x, y, z)$ in Cartesian coordinates. Similarly define $\widetilde{F}(q, \beta, z)=\widetilde{f}(u, v, z)$, with $(u=q \cos \beta, v=q \sin \beta, z=z)$. A further step consists in making respective $(\theta / \beta)$ angular Fourier decomposition of $F(\rho, \theta, z) / \widetilde{F}(q, \beta, z))$

$$
F(\rho, \theta, z)=\sum_{l \in \mathbb{Z}} F_{l}(\rho, z) e^{i l \theta}, \quad \widetilde{F}(q, \beta, z) \sum_{l \in \mathbb{Z}} \widetilde{F}_{l}(q, z) e^{i l \beta} .
$$

Then equation (2) is reduced to an integral equation for the circular components

$$
\begin{gathered}
\frac{1}{\sin \omega K(\omega)}\left(\mathcal{R}_{C} F\right)_{l}(\rho, \omega \mid \zeta)= \\
\int_{0}^{\infty} r d r g(r) \int_{0}^{\infty} q d q 2 \pi J_{0}(2 \pi q r \sin \omega) 2 \pi i^{-l} J_{l}(2 \pi \rho q) \widetilde{F}_{l}(q, \zeta+r \cos \omega),
\end{gathered}
$$

where $(\mathcal{R} F)_{l}$ is the $l^{\text {th }}$-circular component of $\mathcal{R} F$.

Now using Hankel's identity, for $n=0, l$

$$
\int_{0}^{\infty} \xi d \xi 2 \pi J_{n}(2 \pi a \xi) 2 \pi J_{n}(2 \pi b \xi)=\frac{1}{a} \delta(a-b)
$$

one obtains the inverse formula, when $g(r)=A / r^{\nu}$ with $\nu \in \mathbb{R}$, under the form

$$
\widetilde{F}_{l}\left(Q, \zeta+\frac{Z}{Q}\right)=
$$




$$
\frac{Z^{\nu}}{A} Q^{2-\nu} \int_{0}^{\infty} \sigma d \sigma 2 \pi J_{0}(2 \pi \sigma Z) i^{l} G(t) \int_{0}^{\infty} \rho d \rho 2 \pi J_{l}(2 \pi \rho Q)\left(\mathcal{R}_{C} F\right)_{l}(\rho, \sigma \mid \zeta),
$$

where $\omega=\tan ^{-1} \sigma$ with $0<\omega<\pi / 2$ and

$$
G(t)=\frac{\cos ^{2-\nu} \omega}{\sin \omega K(\omega)}
$$

$Z$ and $Q$ are positive real numbers. The last step consists in collecting the circular components to reconstruct the two dimensional Fourier transform $\widetilde{F}(Q, \beta, \zeta+Z / Q)=$ $\widetilde{f}(U, V, \zeta+Z / Q)$ in order to apply the inverse Fourier transform which gives back $f(X, Y, \zeta+Z / Q)$.

Now for back scattering scanning, the procedure is the same but care must be exercised for $\cos \omega<0$. The reader can find the derivation in [15]. As noted before, this inverse formula supports the imaging procedure at right of Fig. 4.

- Inspection shows that, for $\mathcal{R}_{C C}$, the role of $\widetilde{F}_{l}(q, \zeta+r \cos \omega)$ is replaced by

$$
\int_{0}^{\infty} d \zeta \frac{A}{\zeta^{\nu}} \widetilde{F}_{l}(q, \zeta+r \cos \omega)
$$

As the support of $f(x, y, z)$ is in the upper space $z>0$, we may extend the $\zeta$ integration to the full $\mathbb{R}$. Thus the expression of equation (12), is just a convolution where the unknown function is $\widetilde{F}_{l}$. Hence by standard de-convolution, the Fourier transform of $\frac{A}{\zeta^{\nu}}$ being known, one gets back $\bar{F}_{l}(Q, P)$, which is the one dimensional Fourier transform in the last variable $Z$ of $\widetilde{F}_{l}(Q, Z)$, leading indirectly to the reconstruction of $f(x, y, z)$ (for details follow $[8,11]$ ).

Lately, impressive progress has been achieved on cone Radon transforms on cylinder or spherical surfaces by M Haltmeier and co-workers[18]. Several inverse formula for three dimensional Compton cameras reconstruction have been established by S Moon [19, 20], V Maxim et al [21] and P Kuchment and F Terzioglu [22, 23]. They surely will induce further results both useful for integral geometry as well as for next generation imaging processes.

\section{Two-dimensional Compton scattered radiation imaging and the V-line Radon transform}

\subsection{Original proposal}

As no reliable reconstruction procedure was found, in the early 90 's, for various forms of Compton cameras since it was proposed, R Basko, G L Zeng and G T Gullberg in 1997 have had the idea of considering a simpler one dimensional Compton camera, which means that scattering and absorption detectors are linear arrays and which is aimed to be used for two dimensional objects, as shown in Fig. 8. As data is gathered along a two dimensional cone, which is a geometric figure made up of two half lines meeting at a vertex site, these authors have coined the name of $V$-projections and have sought to work out a reconstruction algorithm for the object electron density. A full analytic solution for this one dimensional Compton camera has appeared recently in $[19,25]$.

In 2005, we have considered a one dimensional Compton scatter SPECT (CSSPECT) [26], which uses a Compounded V-line Radon transform, a two dimensional version of the previous Compounded Conical Radon Transform (CCRT), see Fig. 9. 


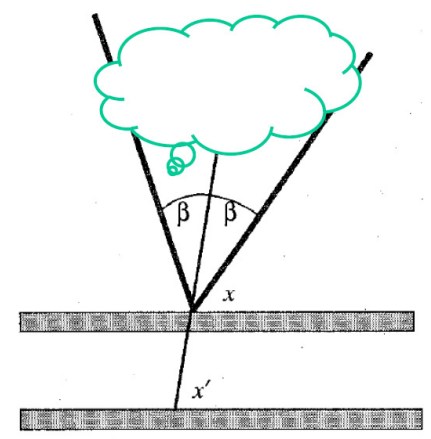

Figure 8. Basko-Zeng-Gulberg Compton camera
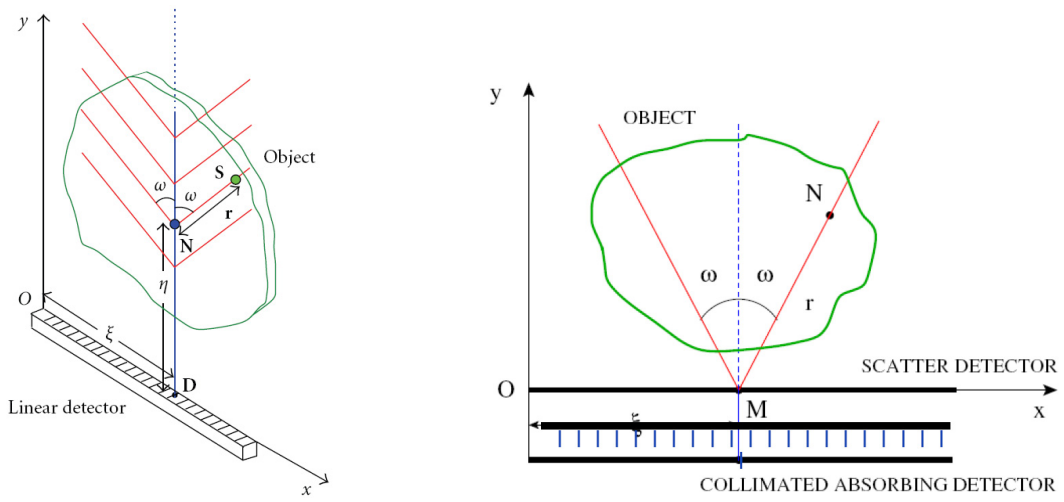

Figure 9. 2D Compton scatter SPECT (left) and 2D Cree-Bones Compton camera (right), reprinted from [27]

- Looking back, we have realized that a two dimensional version of the Cree-Bones Compton camera makes use now of a simple V-line Radon transform, which becomes by its own status an interesting Radon transform worth of a separate study. This has been done also in [27].

Following the outlay of section 4 with the same notations but suppressing one coordinate, we define the V-line Radon Transform of a function $f(x, y)$ supported in the upper half-plane as

$$
\mathcal{V} f\left(x_{D}, \omega \mid \zeta\right)=\int_{\mathbb{R}^{2}} d x d y \mathcal{K}_{V}\left(x_{D}, \omega, \zeta \mid x, y\right) f(x, y),
$$

with

$$
\begin{gathered}
\mathcal{K}_{V}\left(x_{D}, \omega, \zeta \mid x, y\right)= \\
K(\omega) g\left(\frac{y-\zeta}{\cos \omega}\right)\left(\delta\left(\left(x-x_{D}\right) \cos \omega-(y-\zeta) \sin \omega\right)+\delta\left(\left(x-x_{D}\right) \cos \omega+(y-\zeta) \sin \omega\right)\right),
\end{gathered}
$$

where $K(\omega)$ and $g(r)$ have been defined earlier in section 4. The reconstruction formula for scanning angles $0<\omega<\pi / 2$ is a slight modification of the one given in [27] for $\zeta=0$,

$$
f(x, \zeta+z)=
$$


$\frac{1}{2 \pi^{2}} \int_{0}^{\infty} \frac{d \tau}{\overline{K(\tau)} \sqrt{1+\tau^{2}}}$ P.V. $\left\{\int_{\mathbb{R}} d x_{D} \frac{\bar{V} f^{\prime}\left(x_{D}, \tau \mid \zeta\right)}{x_{D}-x-(\zeta+z) \tau}+\int_{\mathbb{R}} d x_{D} \frac{\bar{V} f^{\prime}\left(x_{D}, \tau \mid \zeta\right)}{x_{D}-x+(\zeta+z) \tau}\right\}$.

where $\tau=\tan \omega, \overline{K(\tau)}=K(\omega)$ and $\bar{V} f^{\prime}\left(x_{D}, \tau \mid \zeta\right)=\frac{d}{d x_{D}} V f\left(x_{D}, \omega \mid \zeta\right)$, and $g(r)=1$ for simplicity. It turns out that, although the V-line Radon transform has originated from emission imaging, it will have also application in transmission-reflection imaging, see section 6 .

Finally, coming back to emission imaging, we may have here the two dimensional counter parts of Fig. 5, respectively for scanning angle ranges $0<\omega<\pi / 2$ and $\pi / 2<\omega<\pi$.
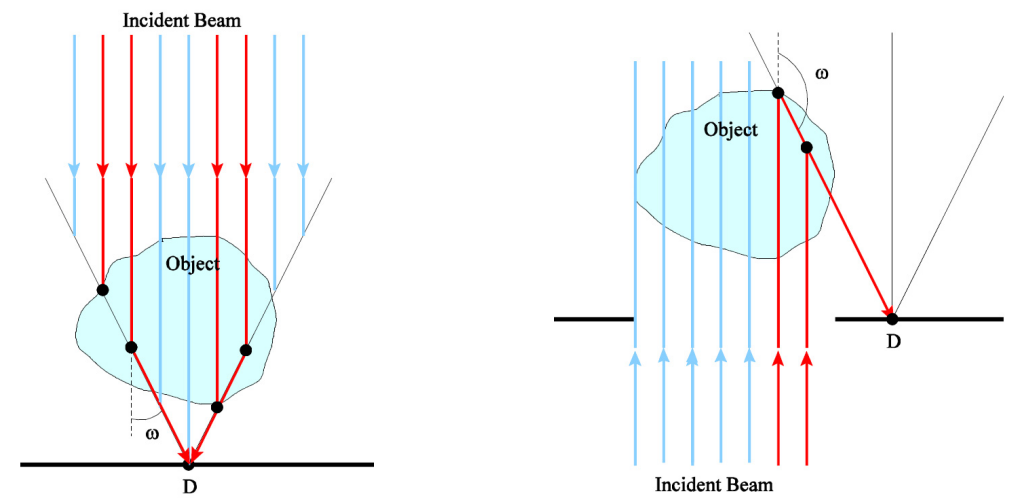

Figure 10. Two dimensional Transmission-Scattering Imaging with parallel beam

- A two dimensional version of emission scattering SPECT imaging can also be considered here analogously to the three dimensional case of section 4 . Image formation is described by the previous $\mathrm{V}$-line Radon transform but $\zeta$-integrated. This compounded $\mathrm{V}$-line Radon transform of a function $f(x, y)$ is given by

$$
\mathcal{V}_{C} f\left(x_{D}, \omega\right)=\int_{\mathbb{R}^{2}} d x d y \mathcal{K}_{C V}\left(x_{D}, \omega \mid x, y\right) f(x, y),
$$

where the kernel is

$$
\begin{gathered}
\mathcal{K}_{C V}\left(x_{D}, \omega \mid x, y\right)= \\
\int_{0}^{\infty} d \zeta g(\zeta) \mathcal{K}_{V}\left(x_{D}, \omega, \zeta \mid x, y\right)=\frac{K(\omega)}{\sin \omega}\left\{g\left(y-\frac{x-x_{D}}{\tan \omega}\right)+g\left(y-\frac{x+x_{D}}{\tan \omega}\right)\right\} .
\end{gathered}
$$

The inverse formula can be worked out as in section 4 . The result of equation (15) remains valid with the data $\bar{V} f^{\prime}\left(x_{D}, \tau \mid \zeta\right)$ replaced by

$$
\int_{0}^{\infty} d \zeta g(\zeta) \bar{V} f^{\prime}\left(x_{D}, \tau \mid \zeta\right)
$$

and the final step is also a de-convolution process as stated in [27]. 


\subsection{Connection to Compton scatter tomography (CST)}

Further properties of the V-line Radon transform have been uncovered recently in [33]. It is well known that under geometric inversion of center $O$ (origin of coordinates) and modulus $q$

$$
x^{\prime}=x \frac{q^{2}}{x^{2}+y^{2}}, \quad y^{\prime}=y \frac{q^{2}}{x^{2}+y^{2}},
$$

the line of equation $x^{\prime}-y^{\prime} \tau-\xi=0$ in the plane $\left(x^{\prime} O y^{\prime}\right)$ is transformed into a circle $(\Gamma)$ of equation $x^{2}+y^{2}-\xi^{\prime} x-\tau \xi^{\prime} y=0$ in the plane $(x O y)$, going through the origin $O$ with radius $\xi^{\prime} \sqrt{1+\tau^{2}} / 2$ and center at $\left(\xi^{\prime} / 2, \xi^{\prime} \tau / 2\right)$, with $\xi^{\prime}=q^{2} / \xi$. This observation has an immediate and simple consequence for integral geometry. The classical Radon transform of a function $f(x, y)$ may be represented by the Cormack circular Radon transform of the function

$$
g\left(x^{\prime}, y^{\prime}\right)=\frac{q^{2}}{x^{\prime 2}+y^{\prime 2}} f\left(x^{\prime} \frac{q^{2}}{x^{\prime 2}+y^{\prime 2}}, y^{\prime} \frac{q^{2}}{x^{\prime 2}+y^{\prime 2}}\right) .
$$

on the circle $(\Gamma)$. Moreover, the Radon transform on lines in an upper half place of $f(x, y)$ with support in this half plane may be expressed as the Cormack circular Radon transform of $g\left(x^{\prime}, y^{\prime}\right)$, not on full circles but on circular arcs located on the corresponding upper half plane and going through the origin of coordinates.

At the beginning of section 4, we have mentioned Compton Scatter Tomography (CST) as another imaging concept based on Compton scattering of ionizing radiation with matter electrons. Norton S J, in [2] was the first to propose a concrete CST modality, in which the collected data is the amount of scattered radiation detected at energy $E(\omega)$ due to all scattering sites located on a circular arc connecting the point source $S$ to the detection point $D$ and subtending an inscribed angle of $(\pi-\omega)$ radians, see e.g. [5]. Under a geometric inversion of center $S$ and arbitrary modulus $q$, this circular arc is mapped onto a half-line in the upper half-plane starting at site $D^{\prime}$ on the line $S D$ such that $S D \cdot S D^{\prime}=q^{2}$. Thus the "Norton Radon circular arc transform" is nicely mapped onto the Radon transform on half-lines of the upper-plane, which was shown to be invertible [33].

So if one applies now the previous geometric inversion to a V-line with vertex on the line $S D$, this V-line will be transformed into a pair of supplementary circular arcs $\ddagger$ passing through the inversion center $S$. Consequently the integral of a function on the V-line becomes the integral of a new function on the double arc. This appears as a generalization of the CST introduced by S J Norton, which has been studied in [33] and shown to be relevant for CST, see Fig. 11

Thus the V-line Radon transform is thus instrumental in providing an inverse formula to a new CST problem using a double scanning procedure which may be considered as an extension of the one proposed long ago by S J Norton.

\subsection{Subsequent generalizations of the V-line Radon transform}

The concept of V-line Radon transform turns out to be very inspiring and has, since 2009 initiated a host of novel associated Radon transforms:

- the broken line transform of Florescu et al [28, 29, 30]. This Radon transform has come up in the investigation in Single-Scattering Optical Tomography. This Radon

$\ddagger$ In the sense that they subtend supplementary inscribed angles. 


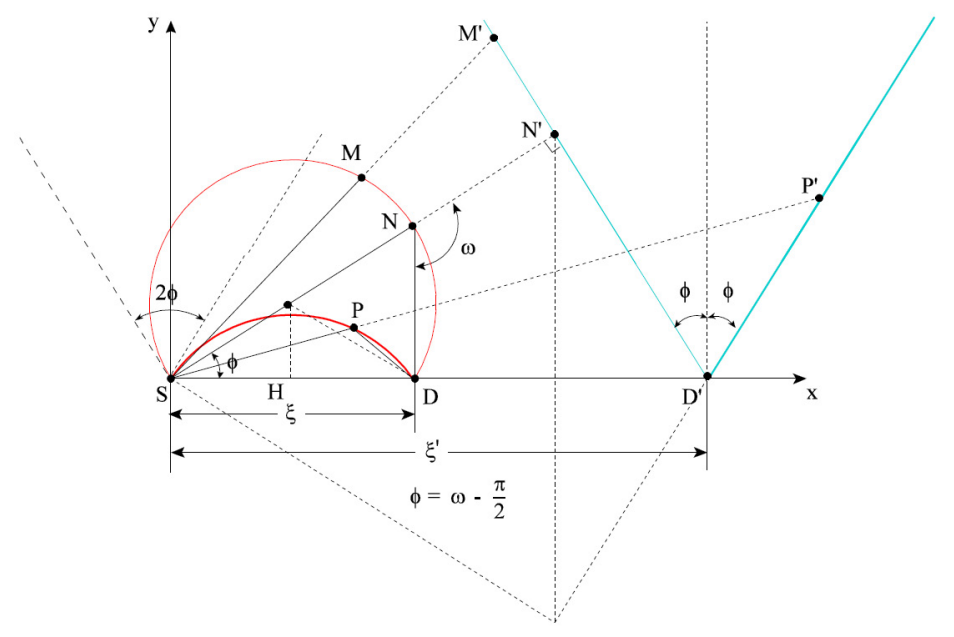

Figure 11. Connection V-line Radon Transform and Double arc CST, reprinted from [33]

transform integrates a function on a V-line shape contour with a constant opening angle and with a movable vertex in a plane.

- the V-line Radon transform on circular path of Moon and Haltmeier [18]. This Radon transform, which integrates a function on a V-line of opening angle $\omega$ inside a circle, with vertex on this circle and with its symmetry axis intersecting the circle center, is shown to have an inverse.

- the "star transform" of Zhao-Schotland-Markel [31]. This Radon transform integrates a function on several half-lines radiating from a movable vertex in the plane, the angular position of each half-line is rigidly fixed with respect to other half-lines.

- Another original generalization is due to Ilmavirta J [32], who shows that Radon transform can be also defined also on a broken line formed by several line segments meeting pairwise at a number of vertices located on a curve in the plane, such that the optical law of reflection on this curve is verified.

\section{Applications}

In this section, we present some simulations illustrating potential applications of the Cone/V-line Radon transforms:

- compounded V-line Radon transform in emission imaging for medical purposes,

- V-line Radon transform in Transmission-Reflection imaging for non-destructive evaluation/inspection,

- Cone Radon transform in Transmission-Scattering imaging for cultural heritage research.

\subsection{Biomedical imaging}

Biomedical imaging is nowadays served almost totally by primary radiation imaging. Overwhelmingly tomographic imaging dominates this domain of application. Here we show simulations that demonstrate the imaging potentiality of Compounded V-line 
Radon Transform in future emission imaging [34]. In Fig. 12, we have displayed an original thyroid phantom, the Compounded V-line Radon Transform data and the reconstructed image of the thyroid. Details of the technical setup of the simulations can be found in [34].
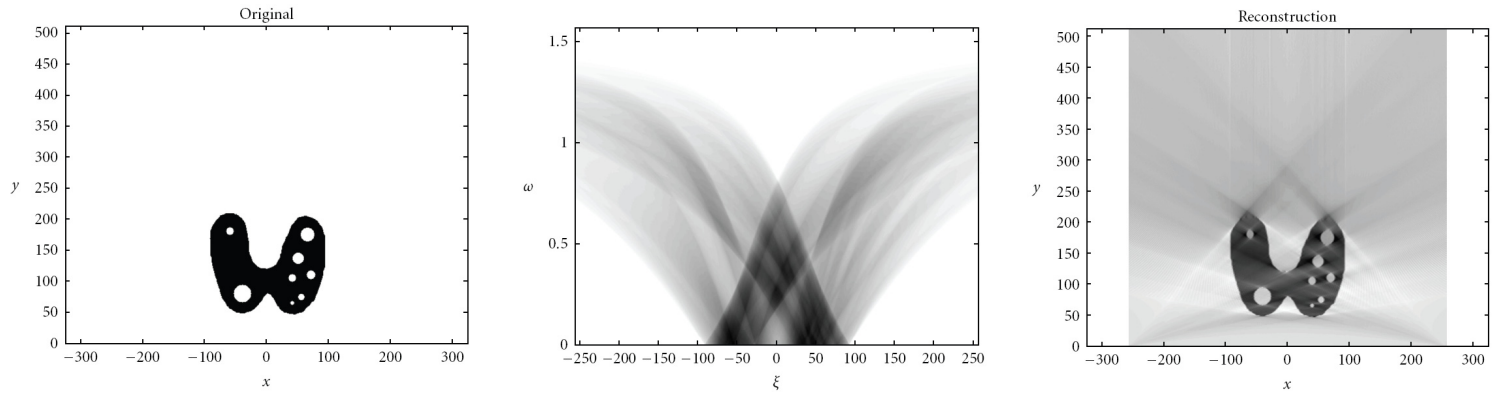

Figure 12. Original medical phantom (left), Compounded V-line Radon transform data (center), Thyroid Reconstruction (right), reprinted from [34]

\subsection{Non destructive Evaluation-Inspection}

A surprising application of the simple V-line Radon transform has arisen in non destructive evaluation-inspection. Instead of considering rectilinear radiation propagation, one may use radiation reflection against appropriate surfaces to obtain propagation along a V-line and collect transmission data along this V-line, see Fig. 13. This is why we have called this imaging process transmission-reflection imaging, see [35]. In Fig. 14, we exhibit an original plastic crack, its V-line Radon transform data and its reconstructed image.
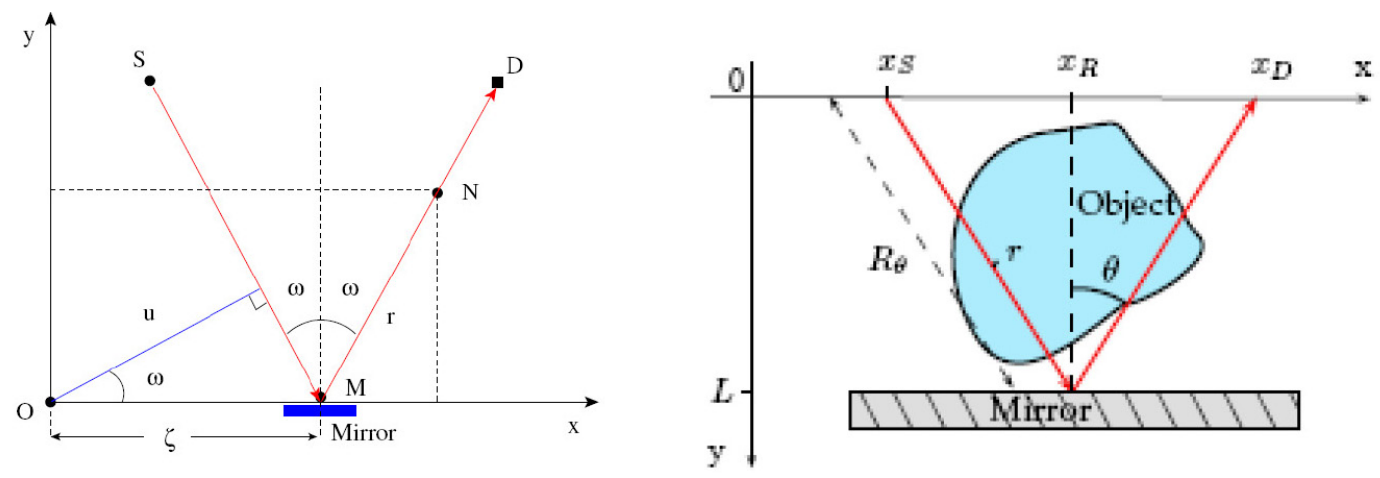

Figure 13. V-line Radon transform in Transmission-Reflection Imaging, reprinted from [35] 
Original plastic crack

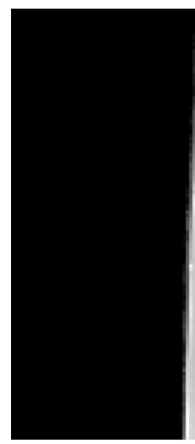

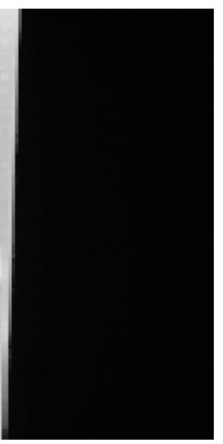

V-line Data
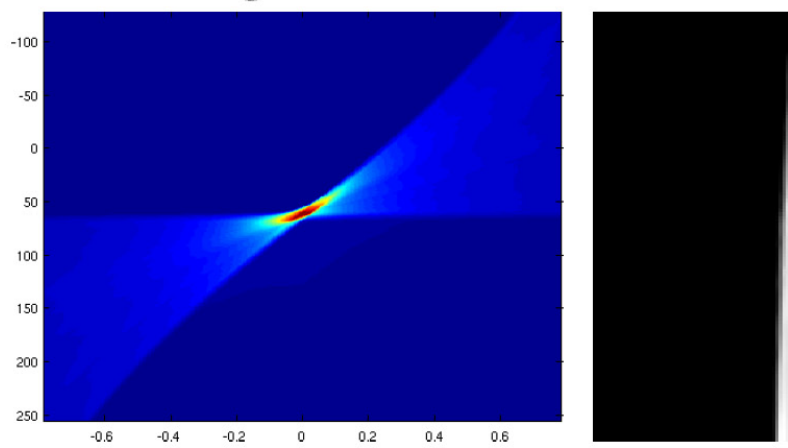

Reconstruction

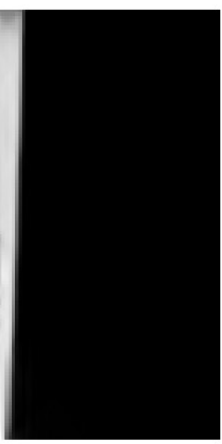

Figure 14. Original plastic crack (left), V-line Radon transform data (center), Reconstruction (right), reprinted from [35]

\subsection{Cultural heritage object imaging}

Cultural heritage research is a new application domain for imaging processes. The need for conservation of cultural heritage treasures has led to investigations into their deep internal structures and compositions. Such a knowledge would indicate which conservation methods are appropriate to guarantee their survival over centuries. Among a plethora of methods, the use of Compton scattered radiation imaging has been advocated recently in [36]. Thus it is natural to test whether scattered imaging processes which rely on Cone / V-line Radon transforms have performances that fit the requirements of cultural heritage research.

Objects under investigation in cultural heritage research are of inestimable value and rarity. They need to be treated with the utmost care even in the case of non invasive inspection or scrutiny. Of course not all of them can be suitable for scattered radiation imaging. Here we show that delicate flat but large objets in cultural heritage research can be handled by three dimensional scattered radiation imaging. The chosen modality is the one shown in the right figure of Fig. 4. This is because the object is unique and of large size. It cannot be manipulated as an industrial object and must be fitted to the radiation source (e.g. a synchrotron beam) from the back, see Fig. 15. Data is then collected according to this setup. The reconstruction algorithm follows the method developed in [37].

Simulations results are presented in Fig. 16 in order to ascertain the viability of this imaging process. A numerical object corresponding to a flat stratigraphic sample is created. Several cross sections of this phantom are represented with its layers, electron densities and some inhomogeneities of random sizes and positions. The reconstruction is performed with the conical Radon transform inversion formula and results are given in Fig. 16. More details may be found in [38, 39].

\section{Conclusion}

Over the last two decades, decisive progress has be achieved in the field of Compton scattering imaging thanks to the invertibility of a family of Radon transform on circular cones. This class of Radon transform in $\mathbb{R}^{3}$, in the so-called compounded 


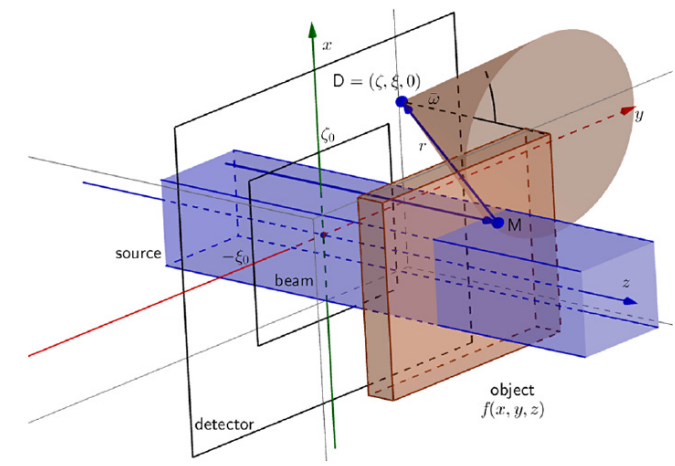

Figure 15. Practical setup for investigating a cultural heritage object using synchrotron radiation beam, reprinted from [39]
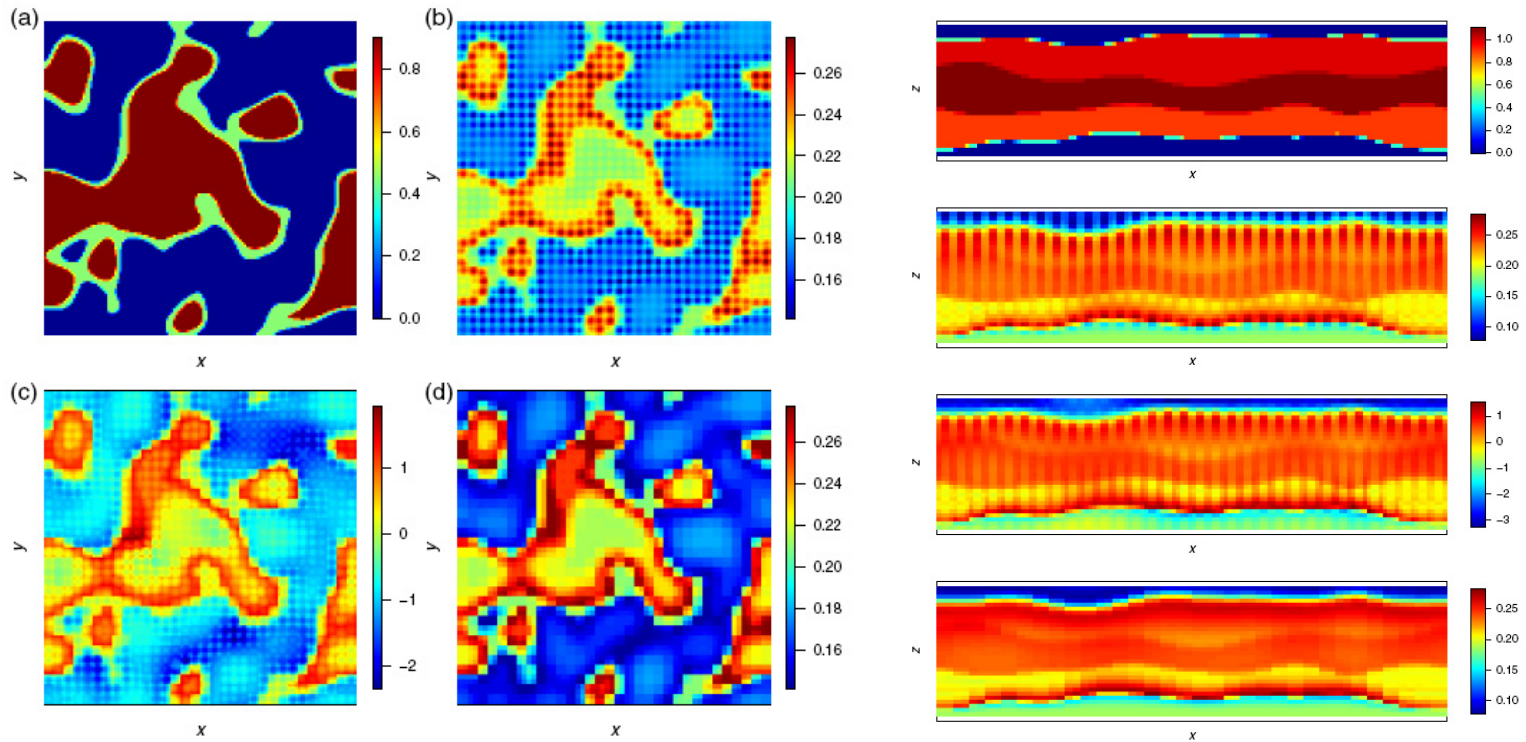

Figure 16. Left - Stratigraphic phantom of dimensions $128 \times 128 \times 32$ voxels. Plane presented: $z=4$. (a) original phantom, (b) direct inversion, (c) inversion after normalizing values following $z$-axis, and (d) inversion after removing voxels located at edges of single reconstructions of dimensions $4 \times 4 \times 32$ voxels

Right - Stratigraphic phantom of dimensions $128 \times 128 \times 32$ voxels. Plane presented: $y=22$. From top to bottom: original phantom, direct inversion, inversion after normalizing values following z-axis, inversion after removing voxels located at edges of single reconstructions of dimensions $4 \times 4 \times 32$ voxels, reprinted from [39]

form, supports a remarkable imaging process known as Compton scatter SPECT imaging which can provide directly the three dimensional image of the interior of an object by a standard gamma camera functioning in a fixed spatial position. The absence of rotational motion characteristic of standard SPECT imaging represents a major advantage resulting from the exploitation of scattered gamma rays for imaging 
purpose. It should be adequate and useful in many situations arising in medical diagnostics as well as in security monitoring.

We have also explore the two dimensional version of this transform, which is known under the name of V-line Radon transform. This concept was a very fruitful concept which was shown to support some particular tomographic imaging (or slice imaging). Most remarkably is an application to a new modality combining transmission and reflection imaging using for example laser light traveling in slightly opaque optical media. A surprising consequence is that it has led to some interesting generalizations such as the "broken line" Radon transform, the star Radon transform and the Radon transform on generalized broken lines with vertices on a smooth curve such that the optical reflection law is verified. Further generalizations can also be expected.

The main interest in these Radon transforms is the existence of analytic inverse formulas which lay foundations for corresponding imaging principles. Numerical simulations under various working conditions are made in order to ascertain their viability and performances. Of course there remains also additional steps to overcome, for example how to include the effects of attenuation [40] and how to insure reconstruction robustness against noise. These issues will be addressed certainly in future work.

Finally it is very gratifying to recognize the utility of mathematical integral geometry for solving practical inverse problems in Compton scatter imaging science.

\section{References}

[1] Cormack A M, Representation of a function by its line integrals with some radiological applications, Jour. Appl. Phys.34:2722-2727 (1963).

[2] Norton S J, Compton scattering Tomography, Jour. Appl. Phys.76(4):2007-2015 (1995).

[3] Nguyen M K, Faye C, Eglin L, and Truong T T, Apparent Image Formation by ComptonScattered Photons in Gamma-Ray Imaging, IEEE Signal Processing Letters8(9):248-251 (2001).

[4] Clarke R L, Milne E N, \& Van Dyk G, (1976). The use of Compton scattered gamma rays for tomography, Invest. Radiol. 11(3):225-235 (1976).

[5] Truong T T and Nguyen M K (2012) Recent Developments on Compton Scatter Tomography: Theory and Numerical Simulations, in "Numerical Simulation - From Theory to Industry", Dr. Mykhaylo Andriychuk (Ed.), INTECH Rijeka Croatia.

[6] Singh M, An electronically collimated gamma camera for single photon emission tomography. Part 1: Theoretical considerations and design criteria, Med.Phys.10(4):421-427 (1983).

[7] Cree M J and Bones P J, Towards Direct Reconstruction from a Gamma Camera Based on Compton Scattering, IEEE Transactions on Medical Imaging13(2):398-407 (1994).

[8] Nguyen M K and Truong T T, On an integral transform and its inverse in nuclear imaging, Inverse Problems 18:265-277 (2002).

[9] Nguyen M K, Truong T T, Driol C and Zaidi H, On a novel approach to Compton scattered emission imaging, IEEE Trans. Nucl. Sci. 56(3):1430-1437 (2009).

[10] Nguyen M K, Truong T T, Morvidone M, Zaidi H, Scattered Radiation Emission Imaging: Principles and Applications International Journal of Biomedical Imaging Volume 2011, Article ID 913893 (2011).

[11] Nguyen M K and Truong T T, On the concept of scattered gamma-ray imaging and the corresponding generalized Radon transforms, ESAIM: PROCEEDINGS, H. Ammari, Ed. 26:65-93 (2009).

[12] Gouia-Zarrad R and Ambartsoumian G, Exact inversion of the conical Radon transform with a fixed opening angle, Inverse Problems30:045007 (2014).

[13] Gouia-Zarrad R, Analytical reconstruction formula for n-dimensional conical Radon transform, Computers and Mathematics with Applications68:10161023 (2014).

[14] Moon S, On the Determination of a Function from Its Conical Radon Transform with a Fixed Central Axis, SIAM Journal on Mathematical Analysis 48(3):1833-1847 (2016). 
[15] Truong T T, Nguyen M K and Grangeat P, Radon transforms on a class of cones with fixed axis direction, Jour. Phys. A: Math. Gen.38:80038015 (2005).

[16] Guerrero P, Cohen S, Nguyen M K and Dumas L, Modelling of a New Compton Imaging Modality for an In-Depth Characterisation of Flat Heritage Objects, Eurosim Conference, Lyon France, June 2016.

[17] Guerrero P, Nguyen M K, Dumas L and Cohen S, Three dimensional imaging of flat natural and cultural heritage objects by a Compton scatter modality, Journal of Electronic Imaging 26(1):011026 (2017).

[18] Moon S and Haltmeier M, Analytic inversion of a conical Radon transform arising in application of Compton cameras on the cylinder, SIAM J. Imaging Sciences 10(2):535-557 (2017).

[19] Jung C Y and Moon S W, Inversion formulas for cone transforms arising in application of Compton cameras, Inverse Problems 31:015006 (2015).

[20] Jung C Y and Moon S W, Exact Inversion of the Cone Transform Arising in an Application of a Compton Camera Consisting of Line Detectors, SIAM J. Imaging Sci., 9(2), 520536 (2016).

[21] Maxim V, Frandes M and Prost R, Analytical inversion of the Compton transform using the full set of available projections, Inverse Problems 25:095001 (2009).

[22] Terzioglu F, Some inversion formulas for the cone transform, Inverse Problems 31:115010 (2015).

[23] P Kuchment $\mathrm{P}$ and Terzioglu F, Three-Dimensional Image Reconstruction from Compton Camera Data, SIAM Journal on Imaging Sciences 9(4):1708-1725 (2016).

[24] Basko R, Zeng G L and Gullberg G T, Analytical Reconstruction Formula for One-Dimensional Compton Camera, IEEE Trans. Nucl. Sci.44(3):1342-1346 (1997).

[25] Hristova Y, Inversion of a V-line transform arising in emission tomography, Journal of coupled systems multiscale dynamics3(3):272-277 (2015).

[26] Nguyen M K and Truong T T, Imagerie par rayonnement gamma diffusé, Hermès Science Publications Paris 2006.

[27] Truong T T and Nguyen M K, On new V-line Radon transforms in $\mathbb{R}^{2}$ and their inversion, Jour. Phys. A: Math. Theor. 44:075206 (2011).

[28] Florescu L, Markel V A and Schotland J C, Inversion formulas for the broken-ray Radon transform Inverse Problems 27:025002 (2011).

[29] Katsevich A and Krylov R, Broken ray transform: inversion and a range condition, Inverse Problems 29:075008 (2013).

[30] Ambartsoumian G and Moon S W, A series formula for inversion of the V-line Radon transform in a disc, Computers and Mathematics with Applications 66:15671572 (2013).

[31] Zhao F, Schotland J C and Markel V A, Inversion of the star transform, Inverse Problems 30:105001 (2014).

[32] Ilmavirta J, Broken ray tomography in the disc, Inverse Problems 29:035008 (2013).

[33] Truong T T and Nguyen M K, New properties of the V-line Radon transform and its imaging applications, Jour. Phys. A: Math. Theor. 48:405204 (2015).

[34] Morvidone M, Nguyen M K, Truong T T and Zaidi H, On the V-line Radon Transform and its imaging applications, International Journal of Biomedical Imaging 2010:208179 (2010).

[35] Régnier R and Nguyen M K, A novel coupled transmission-reflection tomography and the V-line Radon, IEEE-International Conference Image Processing (ICIP), Brussels,September 2011.

[36] Harding G and Harding E, Compton scatter imaging:A tool for historical exploration, Applied Radiation and Isotopes68:9931005 (2010).

[37] Cebeiro J, Morvidone M. and Nguyen M K, Back-projection inversion of a conical Radon transform, Inverse Problems in Science and Engineering 24(2): 328-352 (2016).

[38] Guerrero P, Cohen S, Nguyen M K and Dumas L, Modelling of a new Compton imaging modality for an in-depth characterisation of flat Heritage Objects, Eurosim Conference, Lyon France, June 2016.

[39] Guerrero P, Nguyen M K, Dumas L and Cohen S, Three dimensional imaging of flat natural and cultural heritage objects by a Compton scatter modality, Journal of Electronic Imaging 26(1):011026 (2017).

[40] Novikov R G, On the range characterization for the two-dimensional attenuated x-ray transformation, Inverse Problems 18 (3): 677-700 (2002). 